\title{
Ion heat transport dynamics during edge localized mode cycles at ASDEX Upgrade
}

\author{
E. Viezzer ${ }^{1,2}$, M. Cavedon ${ }^{3}$, E. Fable ${ }^{3}$, F. M. Laggner ${ }^{4,5}$, R. M. \\ McDermott $^{3}$, J. Galdon-Quiroga ${ }^{1,2}$, M. G. Dunne ${ }^{3}$, A. \\ Kappatou $^{3}$, C. Angioni ${ }^{3}$, P. Cano-Megias ${ }^{1,2}$, D. J. \\ Cruz-Zabala $^{1,2}$, R. Dux ${ }^{3}$, T. Pütterich ${ }^{3}$, F. Ryter ${ }^{3}$, E. Wolfrum ${ }^{3}$, \\ the ASDEX Upgrade Team and the EUROfusion MST1 Team $\ddagger$ \\ 1 Dept. of Atomic, Molecular and Nuclear Physics, University of Seville, Avda. \\ Reina Mercedes, 41012 Seville, Spain \\ 2 Centro Nacional de Aceleradores CNA (Universidad de Sevilla, Junta de \\ Andalucia, CSIC), Avda. Thomas A. Edison 7, 41092 Seville, Spain \\ 3 Max Planck Institute for Plasma Physics, Boltzmannstr. 2, 85748 Garching, \\ Germany \\ 4 Institute of Applied Physics, TU Wien, Fusion@ÖAW, Wiedner Hauptstr. 8-10, \\ 1040 Vienna, Austria \\ 5 Dept. of Mechanical and Aerospace Engineering, Princeton University, Princeton, \\ New Jersey, United States \\ E-mail: eviezzer@us.es
}

October 2017

\begin{abstract}
The edge ion heat transport is analyzed in ASDEX Upgrade (AUG) by combining a comprehensive set of pedestal measurements with both interpretive and predictive modelling. The experimentally determined ion heat diffusivities, $\chi_{i}$, are compared with neoclassical theory and the impact of edge localized modes (ELMs) on the edge ion heat transport level is studied in detail. Pedestal matching experiments in deuterium and hydrogen plasmas show that the inter-ELM pedestal $\chi_{i}$ remains close to the neoclassical value. The additional power needed in hydrogen to get similar pedestal temperatures as in deuterium plasmas mostly affects the electron heat channel, i.e. the electron heat diffusivity increases while the ion heat diffusivity stays at the same level within the uncertainties. Sub-ms measurements of the edge ion temperature allows us to extend the analysis to the entire ELM cycle. During the ELM crash, the ion heat transport is increased by an order of magnitude. The perturbed heat flux increases first at the separatrix, i.e. first the separatrix ion temperature increases, leading to a flatter ion temperature gradient, followed by a decrease of the whole pedestal profile. The ion heat transport returns to its pre-ELM neoclassical level 3-4 ms after the ELM crash.
\end{abstract}




\section{Introduction}

Heat transport plays a key role in understanding fusion plasmas. At the transition from L- to H-mode, an edge transport barrier (ETB) for particles and heat is formed and a region with strongly reduced turbulent transport is established. The pedestal with its very strong gradients and proximity to open field lines, requires a different physics treatment than the plasma core. It is widely believed that the shear in the $\mathbf{E} \times \mathbf{B}$ velocity is fundamental for edge turbulence suppression and the formation of the ETB, thus, leading to the transition into H-mode [1].

Comprehensive heat transport studies of the plasma core have been carried out in the past $[2,3,4]$. In this region, the ion thermal transport is usually governed by long wavelength turbulence, such as ITG modes $[5,6]$. The pedestal, with its small spatial width (typically $1.5-2 \mathrm{~cm}$ at ASDEX Upgrade, AUG) and fast temporal changes due to ELMs, is more challenging to analyze. Therefore, comparatively little work has been done in this region. At AUG substantial effort has been put into upgrading the diagnostics at the plasma edge to resolve the fast dynamics and the steep gradients of the pedestal. The unique edge diagnostic suite now available at AUG allows us to measure the edge plasma profiles on a sub-ms to ms timescale with a spatial resolution of better than $5 \mathrm{~mm}$ [7]. Previous AUG experiments indicated that the pre-ELM pedestal ion heat diffusion is close to the neoclassical level, at both low and high collisionality $[8,9]$. The electron heat diffusivity is also at the ion neoclassical heat diffusion level, but remains significantly above the electron neoclassical heat diffusivity, consistent with observations at JT-60U [10]. Similarly, a transport analysis of a high- $T_{e}$ electron cyclotron heated plasma in LHD showed that while the measured electron heat flux exceeds the neoclassical values by an order of magnitude, the measured ion heat flux is comparable to the neoclassical prediction [11].

In this paper, we compare the experimentally determined ion heat diffusivity at the plasma edge to neoclassical predictions calculated with the NEOART code [12]. The analysis of the ion heat transport presented in [8] was done in D. Here, we extend the analysis also to hydrogen plasmas and directly compare the pedestal heat transport in $\mathrm{H}$ and $\mathrm{D}$ via pedestal matching experiments. The isotope effect describes the change in confinement observed in plasmas with different main ion species. Deuterium plasmas show improved confinement compared to hydrogen $[13,14]$, contrary to the predictions of the gyro-Bohm scaling. Previous analysis focusing on the L-H transition showed that, for similar ion temperature gradients, the edge ion heat flux at the H-mode transition is about twice as high in $\mathrm{H}$ than in $\mathrm{D}$ [15]. Moreover, a comparison in the H-mode phase showed that in hydrogen the total heating power has to be more than doubled and the applied gas puff increased by an order of magnitude to match the D pedestal parameters [16]. This suggests that $\mathrm{H}$ plasmas typically feature lower electron density gradients, owing to the increased particle transport, while the total heat flux across the pedestal is twice that in $\mathrm{D}[17,16,18]$. As more gas and power are required in $\mathrm{H}$ to match the $\mathrm{D}$ temperature and density pedestals, the total heat and particle fluxes in $\mathrm{H}$ 
are also larger, implying higher heat and particle diffusivities. In this work we further explore the differences in the pedestal heat transport between $\mathrm{D}$ and $\mathrm{H}$ plasmas.

In addition, we have also extended our analysis to the entire ELM cycle, in both deuterium and helium plasmas, utilizing the new edge CXRS spectrometer [19]. Previous AUG studies on the electron dynamics during the ELM cycle showed that the electron density and temperature pedestals have different recovery times [20]. Recently, it was found that the re-establishment of the pedestal top electron pressure after the ELM crash is correlated with the onset of high frequency magnetic fluctuations [21, 22]. The instabilities driving these fluctuations may limit the pedestal gradients prior to the crash. In this work, we add to the picture by measuring in detail the evolution of the ion heat transport during the ELM cycle.

This paper is structured as follows: Section 2 briefly describes the experimental setup. Section 3 presents the analysis methods used in this work. A comparison of the heat fluxes and the ion heat transport coefficients obtained in hydrogen and deuterium pedestal matching experiments is discussed in section 4. Section 5 extends the ion heat transport analysis to the entire ELM cycle, which allows us to study the dynamics of the heat transport before and after the ELM crash. A summary of the presented work is given in section 6 .

\section{Diagnostic setup}

The experiments presented in this paper were performed in the AUG tokamak. AUG is equipped with a high spatial and temporal resolution edge diagnostic suite. The comprehensive set of charge exchange recombination spectroscopy (CXRS) diagnostics $[23,24]$, which provides measurements of the ion temperature, flow velocity and impurity density, is essential for the present work. The edge system was recently upgraded to increase the number of lines of sight (LOS), from 8 in both the toroidal and poloidal directions to 31 and 22, respectively [19]. The edge LOS can be divided between the different spectrometers of the edge CXRS suite. The upgraded CXRS diagnostic now enables fully resolved radial profiles without the necessity of a radial plasma sweep. The standard edge CXRS systems have a temporal resolution of $2.3 \mathrm{~ms}$ and image 24 toroidal LOS and 13 poloidal LOS using two Czerny-Turner like spectrometers [23]. A subset of the 53 edge lines of sight (up to max. 9 LOS) can be connected to a newly designed spectrometer [19] that can be operated with $50 \mu$ s integration times. In order to guarantee a high signal-to-noise ratio at such high time resolution, impurity seeding is required. For the discharges presented in section 5, nine LOS were measured with the fast edge CXRS spectrometer. The upgraded diagnostic now allows us to analyze the behaviour of the impurity density, temperature, flow and $E_{r}$ profiles during fast transient events, such as the L-H transition [25] and ELM cycles [26].

Information on the electron temperature and density profiles is also needed for a complete heat transport analysis. The electron temperature, $T_{e}$, profiles are measured via the electron cyclotron emission [27] and the Thomson scattering diagnostic [28], 
which also provides information on the electron density, $n_{e}$. Additional information on $n_{e}$ is provided by the Lithium beam [29] and interferometry system [30]. The measurements from all of these diagnostics are combined within the integrated data analysis algorithm based on Bayesian probability theory [31] to produce a consistent set of $n_{e}$ and $T_{e}$ profiles on a unified time base.

For all discharges the radial profile alignment technique [32], which is based on the twopoint model [33] and which localizes the profiles with respect to the separatrix position, was applied to the measured profiles. This procedure allows us to reduce the radial uncertainties due to the magnetic equilibrium down to $2-3 \mathrm{~mm}$ [34].

\section{Analysis of energy transport}

The ion and electron heat fluxes, $Q_{i}$ and $Q_{e}$, are determined by the radial component of the energy balance equation for the main ions and electrons. The heat fluxes are obtained by volume integrating the energy sources and losses for a particular channel, $Q_{j}=\int P_{j} \mathrm{dV}$ ( $j$ being ions or electrons). For the ions we include neutral beam injection (NBI), the electron to ion heat flux, and the power that is lost via atomic processes including charge exchange with background neutrals, ionization, and recombination, i.e. $P_{i}=P_{i}^{N B I}+P_{e i}+P_{i}^{a t o m i c}$. For the electrons, additional terms such as the absorbed ECRH power, the ohmic power, and radiation cooling play a role, i.e. $P_{e}=P_{e}^{N B I}+$ $P_{e}^{E C R H}+P_{o h}-P_{r a d}-P_{e i}-P_{e}^{a t o m i c}$. The heat diffusivity, $\chi_{j}(\rho)=-\frac{Q_{j}}{n_{j}} \frac{\partial T_{j}}{\partial \rho}$, is determined by the heat flux $Q_{j}$, the particle density $n_{j}$ and the radial temperature gradient.

In this study, $Q_{i}$ and $Q_{e}$ are provided by time-dependent power balance analysis using the ASTRA code [35]. Here, $n_{e}, T_{e}, T_{i}$, the effective charge $\left(Z_{\text {eff }}\right)$, and $P_{\text {rad }}$ are used as inputs. In addition, the auxiliary power to the ions and electrons, the plasma current, toroidal magnetic field and loop voltage are provided. The minor and major radius, the elongation and triangularity, and the shape of the separatrix determined by a $2 \mathrm{D}$ magnetic equilibrium reconstruction are used. ASTRA reconstructs the equilibrium with this prescribed boundary condition and a 3-moment approach [35]. Note that the temporal variation in the plasma energy is taken into account in the evaluation of the heat flux and the heat diffusivity.

ASTRA can be run in an interpretive or in a predictive way. Using the interpretive approach, the heat diffusivities are determined from $Q_{i}$ and $Q_{e}$ and the experimental input profiles. The necessary transport is inferred by adjusting the heat diffusivities to match the evolution of the measured profiles. In the predictive approach, the heat fluxes are calculated from the energy sources, and the heat diffusivities are prescribed in space and time. The heat transport equations are then solved iteratively for $T_{e}$ and $T_{i}$. The ability of the chosen model (the prescribed diffusivities) to reproduce the profile dynamics can be tested by comparing the evolution of the modelled and measured profiles. When running ASTRA predictively, it is not possible for any single set of diffusivities to reproduce both the ELM and inter-ELM phases, due to the highly anomalous heat fluxes during the crash. Therefore, for all predictive runs shown in this 


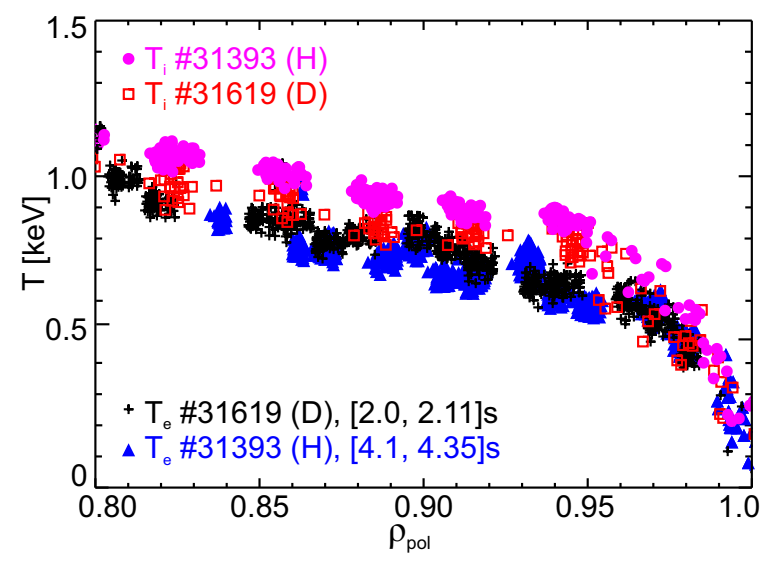

Figure 1. Electron and ion temperature profiles for deuterium and hydrogen.

work the heat diffusivities during the ELM crash are artificially increased to reproduce the behaviour during the ELM crash and the correct starting conditions for the postELM evolution. For the ELM crash itself, non-linear MHD modelling is required to understand and reproduce the observed high anomalous heat transport.

\section{Characterization of the pre-ELM ion heat transport in the edge pedestal - comparison between D and $H$ plasmas}

Pedestal matching experiments in deuterium and hydrogen plasmas were carried out in AUG to study and compare the pedestal structure and stability [16]. Dedicated transport analyses with ASTRA have been performed to analyze the heat fluxes. Figure 1 shows the electron and ion temperature profiles at the plasma edge for both deuterium ( $T_{e}$ in black crosses, $T_{i}$ in red squares) and hydrogen $\left(T_{e}\right.$ in blue triangles, $T_{i}$ in magenta circles) plasmas. The experiments were performed at a toroidal magnetic field, $B_{t}$, on-axis of $-2.5 \mathrm{~T}$, plasma current, $I_{p}$, of $1 \mathrm{MA}$ and an average triangularity of 0.22 (average being the mean of the upper and lower triangularity). To match the density and temperature pedestals, different gas puffs and NBI heating powers were applied [16]. In $\mathrm{D}$, an NBI heating power of $2.5 \mathrm{MW}$ and a D puff of $1.5 \times 10^{21} \mathrm{~s}^{-1}$ were applied, whereas in $\mathrm{H}$, the NBI heating power was increased to $6 \mathrm{MW}$ and the gas puff to $12.1 \times 10^{21} \mathrm{~s}^{-1}$. In both cases, the ECRH heating was $1.1 \mathrm{MW}$. The ion collisionality, defined as the effective collision frequency normalized to the trapped particle bounce frequency [36], in the pedestal (at $\rho_{\text {pol }}=0.97$ ) was 0.53 in $\mathrm{H}$ and 0.49 in $\mathrm{D}$. As shown in figure 1, the edge profiles are very similar, though $T_{i}$ in hydrogen is slightly higher.

The heat transport coefficients are determined using power balance analysis with ASTRA. To this end, $T_{i}, T_{e}$ and $n_{e}$ as well as the radiated power and the effective charge, $Z_{\text {eff }}$, are used as inputs to the analysis. As described in section 3 , the electron and ion heat fluxes are determined by the respective energy sources. Figure 2 shows (a) the fitted profiles of $T_{i}$ and $T_{e}$, (b) the ion and electron energy sources, $P_{i}$ and $P_{e}$, together with the heat exchange rate between electrons and ions, $P_{e i}$, and (c) the resulting heat 

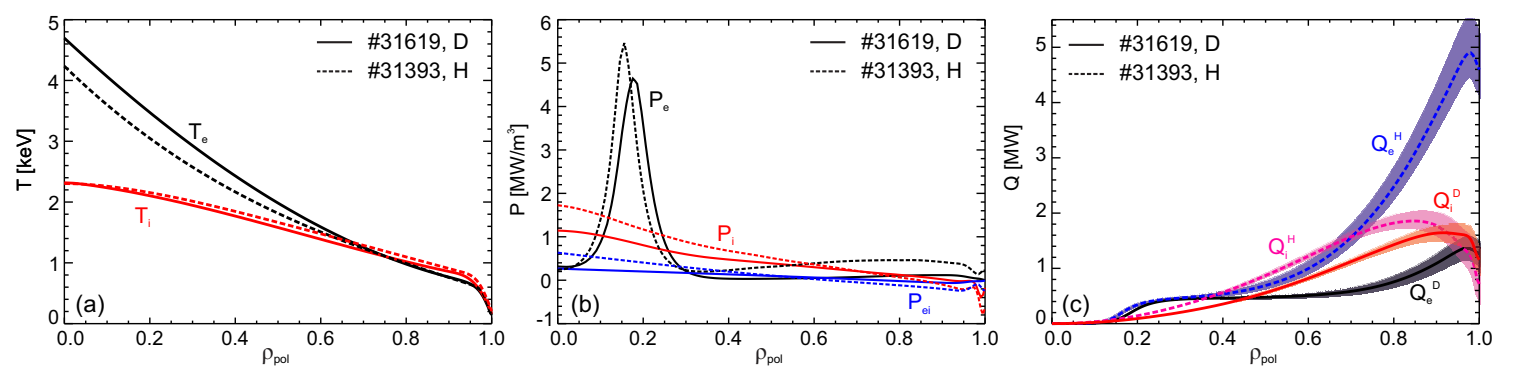

Figure 2. (a) Fitted $T_{i}$ (red) and $T_{e}$ (black) profiles, (b) ion (red) and electron (black) energy sources and electron-ion heat exchange rate (blue), (c) surface-integrated heat fluxes of ions (red, magenta) and electrons (black, blue). Solid lines correspond to the deuterium discharge, while dashed lines to the hydrogen discharge.
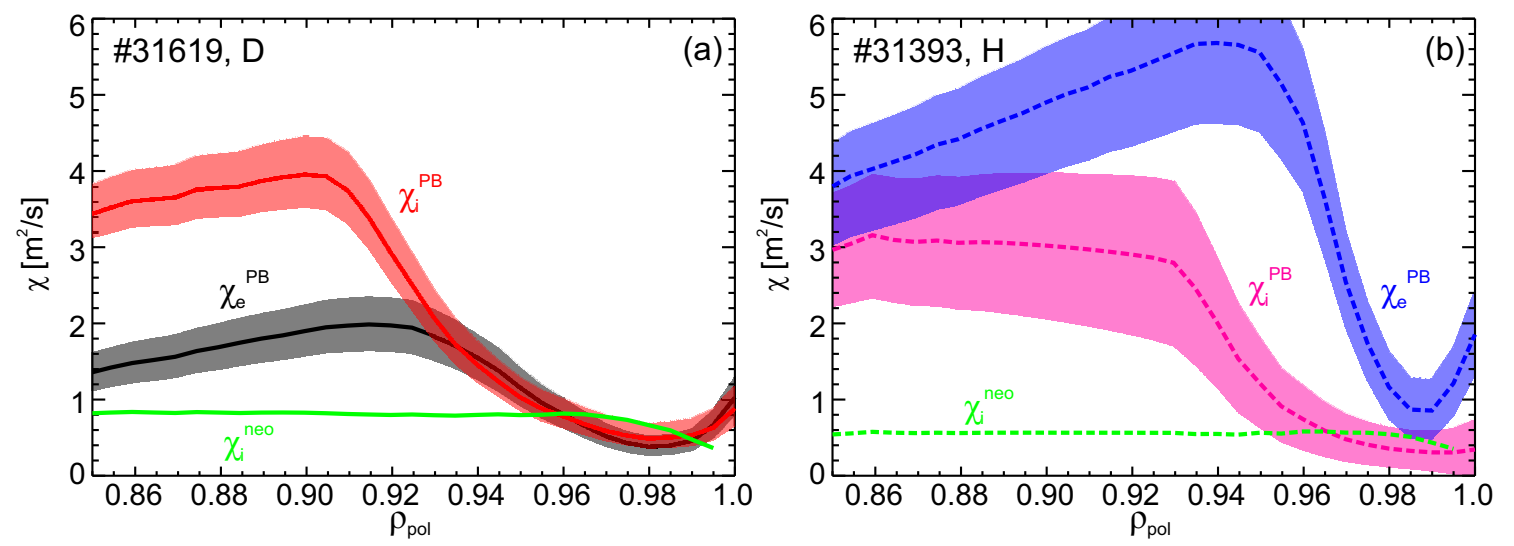

Figure 3. Edge ion heat diffusivities for (a) deuterium and (b) hydrogen.

fluxes. Note that effects due to ELMs have been excluded. The profiles have been averaged over the time window $[-4.5,-1.5] \mathrm{ms}$ before the ELM onset. Due to the mass dependence of the electron-ion heat exchange rate, $P_{e i}$ in hydrogen is approximately a factor of 2 larger than in deuterium [37].

Figure 3 shows the corresponding heat transport coefficients determined via power balance, $\chi_{i}^{P B}$ and $\chi_{e}^{P B}$. At the edge $\left(\rho_{\text {pol }}>0.96\right)$, the ion heat transport is close to the neoclassical level for both $\mathrm{D}$ and $\mathrm{H}$, consistent with previous observations in $\mathrm{D}$ $[9,8]$. Note that the much larger $Q_{e}$ needed in $\mathrm{H}$ (see figure $2(\mathrm{c})$ ) to maintain the same $T_{e}$ means much higher transport in the electron channel. The electron heat diffusivity is higher by a factor of 2-3 in $\mathrm{H}$ compared to $\mathrm{D}$, while the change in the ion heat transport is small (compare figure 3(a) and (b)). In $\mathrm{H}$, the NBI effectively heats the electrons more and thus, the surface-integrated electron heat flux is increased. In order to maintain the same pedestal a higher heat flux in the electron channel is needed, suggesting that the turbulent electron heat transport is higher in $\mathrm{H}$ compared to D.

Note that the neoclassical ion heat diffusivity depends on $\chi_{i}^{\text {neo }} \propto n_{i} \rho_{i, p o l}^{2} / \tau_{i} \propto \sqrt{m}$, where $n_{i}$ is the ion density, $\rho_{i, p o l}$ the poloidal ion gyro-radius, $\tau_{i}$ the ion collisional time, and $m$ the mass. Therefore, the neoclassical $\chi_{i}$ in $\mathrm{H}$ is slightly smaller than in $\mathrm{D}\left(0.6 \mathrm{~m}^{2} / \mathrm{s}\right.$ compared to $0.8 \mathrm{~m}^{2} / \mathrm{s}$ ). This small difference is difficult to resolve in the experiment 
due to the uncertainties. In both cases the neoclassical $\chi_{i}$ is within the experimental uncertainties. It is, however, interesting to note that in the pedestal region $\left(\rho_{\text {pol }}>0.96\right)$, the experimental $\chi_{i}$ does in fact show a decrease by $35 \%$ in $\mathrm{H}$ compared to $\mathrm{D}$, consistent with the expectations from neoclassical theory. Note that the error bars given in figure 3 include uncertainties in the temperature and density profiles as well as uncertainties in the radiated power.

In the following, we extend our analysis to the entire ELM cycle. These measurements were performed utilizing the new edge CXRS spectrometer [19] in a deuterium [26] and a helium plasma.

\section{Evolution of ion heat transport during the ELM cycle}

\subsection{Investigations in deuterium}

The recent upgrade of the edge CXRS diagnostics [19] allows us to measure the dynamics of the ion temperature at AUG with an unprecendented time resolution down to $50 \mu \mathrm{s}$. A dedicated type-I ELMy H-mode discharge was performed to measure the evolution of the ion temperature, impurity density and flows, and the edge radial electric field [26]. Here, $I_{p}$ was $0.8 \mathrm{MA}, B_{t}$ was $-2.5 \mathrm{~T}$, the average triangularity was 0.27 , the core lineaveraged density was $6.9 \times 10^{19} \mathrm{~m}^{-3}$, and the total input power was $5.8 \mathrm{MW}(4.8 \mathrm{MW}$ NBI, 1 MW ECRH). The ELM frequency was constant at around $85 \mathrm{~Hz}$. For the analysis the data measured during the time window $[3.5,5.5] \mathrm{s}$ are synchronized with respect to the onset of the ELMs. In this discharge helium seeding was applied to provide fast CXRS measurements. The measurements were taken on the $\mathrm{He}^{2+}$ spectral line $(n=4 \rightarrow 3, \lambda=468.571 \mathrm{~nm})$ with a temporal resolution of $65 \mu$ s. Note that the ion temperature can be approximated by the measured impurity temperature as the energy equilibration time between main ions and impurities is short (several $\mu \mathrm{s}$ ) compared to local transport time scales $(\sim 1 \mathrm{~ms})$ [34].

Comparison of the ion and electron profiles [26] shows that the ion temperature gradient recovers on a faster timescale than the electron temperature gradient. The maximum $T_{i}$ gradient recovers to its pre-ELM values approximately $4 \mathrm{~ms}$ after the ELM onset, while the maximum $T_{e}$ gradient takes $7-8 \mathrm{~ms}$ [26]. The ion heat transport modelling workflow using ASTRA was applied to the entire ELM cycle to reveal the nature of the ion heat transport dynamics before the ELM crash and during the recovery phase. During an ELM, the plasma moves inward by $5-10 \mathrm{~mm}$, which affects the measurements since different local radial positions are being probed after the ELM onset. This effect was taken into account by mapping the measurements onto the radial coordinate $\rho_{\text {pol }}$ using a high-resolution magnetic equilibrium reconstruction $(100 \mu \mathrm{s})$. Note that the same procedure was applied in the He discharge. In order to have concurrent measurements of $T_{i}, T_{e}$ and $n_{e}$ for the simulations, the data of the fast edge CXRS system was rebinned to $100 \mu \mathrm{s}$ to match the time basis of the $n_{e}$ measurements. As the full profile of $T_{i}$ is needed for the simulations, the measurements of the fast edge CXRS system (CNR) were 


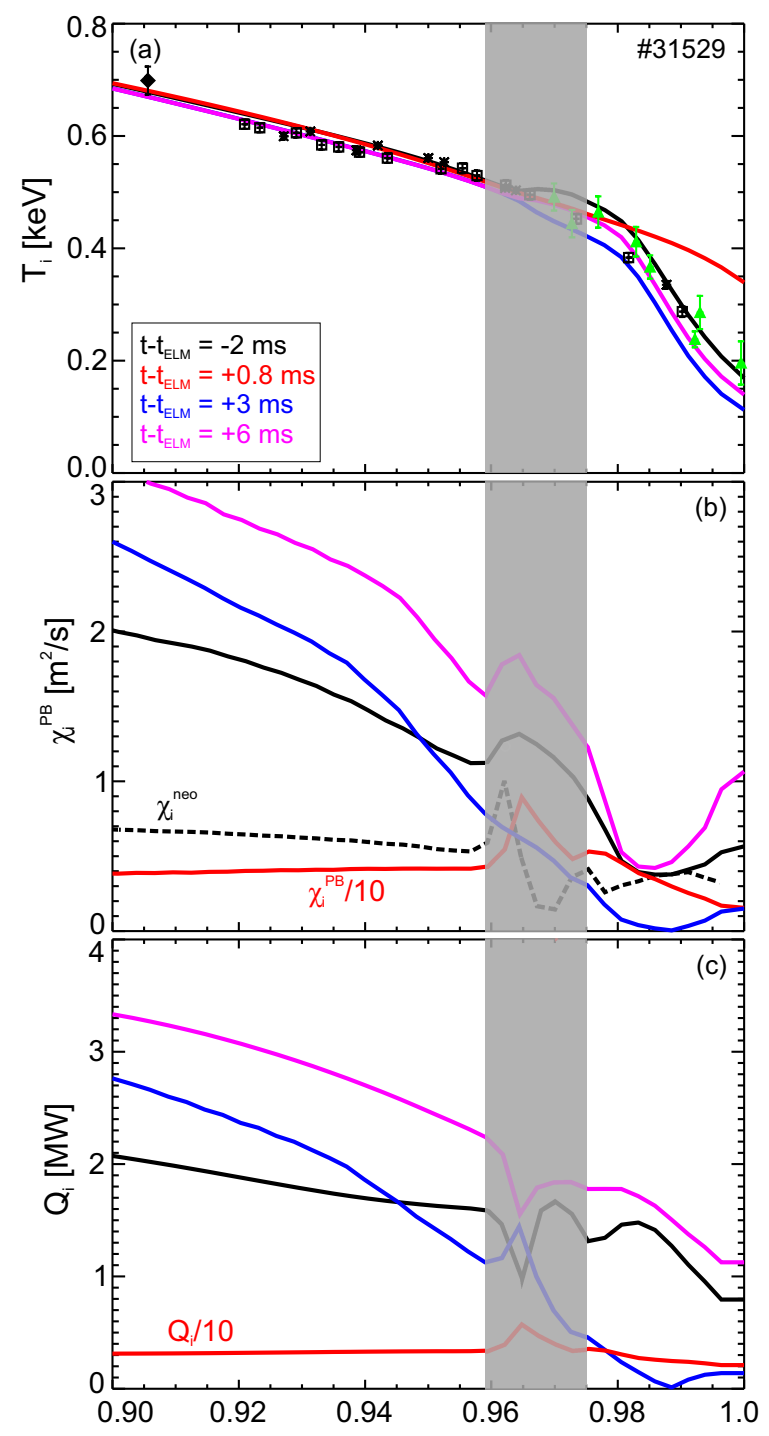

Figure 4. Profiles for four time points during the ELM cycle: (a) $T_{i}$, (b) $\chi_{i}$ determined via power balance and $\chi_{i}^{\text {neo }}$ calculated from neoclassical theory, (c) heat flux $Q_{i}$.

combined with the slower standard edge CXRS diagnostics (CMR and CPR, temporal resolution $2.3 \mathrm{~ms}$ ), also measuring on $\mathrm{He}^{2+}$, and the core CXRS system (CER, set to $5 \mathrm{~ms}$ in this discharge) measuring on $\mathrm{B}^{5+}(n=7 \rightarrow 6, \lambda=494.467 \mathrm{~nm})$. In figure 4 , an example profile measured during the analyzed time window is shown, highlighting the measurement regions of each diagnostic. Here, the measured data points correspond to a pre-ELM profile. Note, these data points do not correspond directly to any of the fitted $T_{i}$ profiles shown. The ELM synchronized measurements of the slower systems have been fitted for various time windows during the ELM cycle and mapped onto the $100 \mu \mathrm{s}$ time basis by interpolating the profiles. Any impact of the ELMs on the preand post-ELM profiles was excluded. For the core system the ELM averaged profile is used due to the limited time resolution. At the overlap point between the slower and fast edge CXRS systems (highlighted in gray in figure 4), the gradients are not real as 
the profiles are interpolated to generate one combined core and edge profile at the fast temporal resolution. The analysis below, therefore, focuses only on the radial region between 0.98 and 1.0.

Figure 4 shows (a) the ion temperature profiles, (b) the ion heat diffusivities and (c) the surface-integrated ion heat flux $Q_{i}$ for four time points during the ELM cycle. Note that the $\chi_{i}$ and $Q_{i}$ profiles highlighted in red are taken during the ELM crash (at time $\mathrm{t}_{\mathrm{t}} \mathrm{t}_{E M}$ $=+0.8 \mathrm{~ms}$ ) and have been divided by a factor of 10 . The perturbation of $T_{i}$ caused by the ELM induces large changes in the heat flux and in the heat diffusivity at the edge. Note that the NBI source stays constant throughout the analyzed time window. The ELM causes large changes in the transport which affect the heat and particle fluxes, leading to flatter temperature and density gradients. In figure 4(b) the neoclassical $\chi_{i}$ profile is highlighted by a dashed line. For clarity, only the profile of the first time point (pre-ELM) is shown. During the ELM cycle, the neoclassical $\chi_{i}$ at the edge increases by a factor of 2 , however, as shown below, the neoclassical flux cannot account for the high ion heat flux observed during the ELM crash. In the core, turbulent transport dominates leading to a strong deviation of $\chi_{i}$ from neoclassical theory, while in the edge transport barrier $\chi_{i}$ approaches the neoclassical level (except for the time points during the ELM). Note that close to the separatrix, the ordering of standard neoclassical theory breaks down and cannot be applied to the region $\rho_{\text {pol }}>0.997$.

Figure 5 shows the evolution of (a) $T_{i}$ and (b) the ratio between the experimentally determined $\chi_{i}^{P B}$ and the neoclassical $\chi_{i}^{\text {neo }}$ during the ELM cycle, as well as (c) the thermo-currents at the inner divertor used as an ELM monitoring signal. At the ELM onset, the separatrix $T_{i}$ increases [26], leading to a reduced gradient in the pedestal, similar to observations at DIII-D [38]. Shortly after the initial separatrix increase, the whole profile drops and then the $T_{i}$ pedestal starts to build up again. The pre-ELM profile is fully recovered $3-4 \mathrm{~ms}$ after the ELM crash, which is faster than the thermal energy confinement time (60 $\mathrm{ms}$ in this discharge).

As shown before, the pre-ELM values of $\chi_{i}$ at the plasma edge are in agreement with neoclassical predictions while, during the crash, $\chi_{i}$ is increased due to anomalous transport caused by the ELM itself. Note that later in the ELM cycle (t- $\left.\mathrm{t}_{E L M}>6 \mathrm{~ms}\right), \chi_{i}$ at the very edge of the plasma is higher than the neoclassical calculation (see figure 5(b)), indicating that close to the separatrix anomalous transport may become important. It should also be noted that during the inter-ELM phase, the uncertainties of the separatrix $T_{i}$ are large due to uncertainties in the equilibrium mapping and in the profile alignment (2-3 mm), and due to a low signal-to-noise ratio. Extending the CXRS measurements into the SOL and combining them with other SOL diagnostics capable of measuring $T_{i}$ would help to quantify $\chi_{i}$ at the separatrix and beyond. This is subject of ongoing work.

The temperature profiles have also been modelled using ASTRA in a predictive way. As described in section 3 , for the predictive approach, the $\chi_{i}$ and $\chi_{e}$ profiles are prescribed and the heat transport equations are solved for $T_{i}$ and $T_{e}$. The $\chi_{i}^{P B}$ profile determined via power balance analysis is used for the plasma core, while at the edge $\left(\rho_{\text {pol }}>0.98\right)$ 


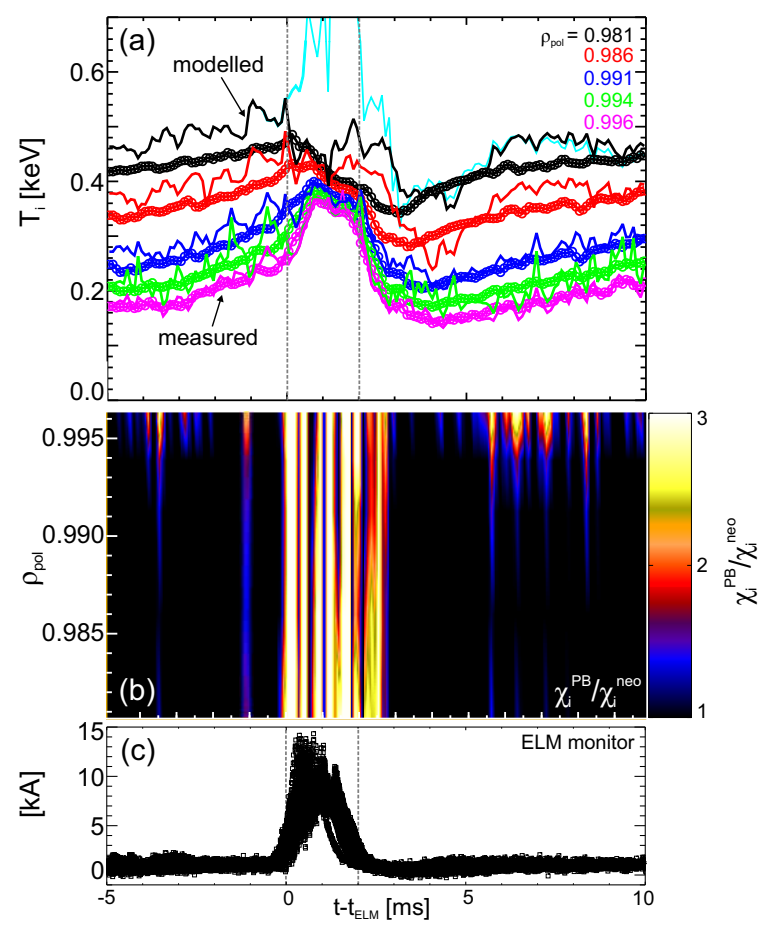

Figure 5. Temporal evolution of (a) $T_{i}$ and (b) $\chi_{i} / \chi_{i}^{n e o}$ during the ELM cycle. (c) Thermo-currents of inner divertor used as ELM monitor. The cyan curve represents $T_{i}$ at $\rho_{\text {pol }}=0.981$ and corresponds to a simulation, in which the edge $\chi_{i}$ during the ELM was increased by a constant factor.

the neoclassical value is used. At the very edge of the plasma $\left(\rho_{\text {pol }}>0.997\right)$, where the neoclassical ordering breaks down, $\chi_{i}^{P B}$ is used. The ELM is simulated by increasing $\chi_{i}$ to an anomalous value at the edge of the plasma. Note that the assumption of a purely diffusive ion heat flux during the ELM crash is probably not $100 \%$ correct as there is also, at the very least, a particle loss across the separatrix. However, for the purposes of a simple model for describing the overall change in magnitude of the transport, a purely diffusive ansatz is used. To obtain good agreement with the temporal behaviour of $T_{i}$, the edge diffusivity profile $\left(\rho_{\text {pol }}>0.90\right)$ is scaled by a linear function, multiplying $\chi_{i}$ by 100 at $\rho_{\text {pol }}=0.9$ and decreasing to 10 at the separatrix, for the time window $[-0.3$, 1.5] $\mathrm{ms}$ with respect to the ELM onset. As the time derivative of the plasma energy matters for the heat flux, the choice of $\chi_{i}$ during the ELM also has an effect on later time points. Simply increasing the edge $\chi_{i}$ during the ELM leads to an overprediction of $T_{i}$ (see cyan curve in figure 5 as an example). The resulting modelled $T_{i}$ profiles are shown in figure 5(a). Note that a time-dependent boundary condition for $T_{e}$ and $T_{i}$ at the separatrix is applied, i.e. the measured values of $T_{e}$ and $T_{i}$ at the separatrix are used. During the inter-ELM phase, good agreement with the experimental values (circles) is obtained, both before the ELM crash and during the recovery phase after the ELM ( $\left.\mathrm{t}-\mathrm{t}_{E L M}>3 \mathrm{~ms}\right)$. The dynamics of the edge $T_{i}$ profile during the pre-ELM phase and during the pedestal build-up after the crash is consistent with neoclassical theory. During the ELM crash itself, the ion heat transport is highly anomalous and would 
require non-linear MHD modelling to understand the behaviour.

\subsection{Analysis in a helium plasma}

Helium plasmas can be used to obtain a direct measurement of the main ion temperature, density and flow by using CXRS on $\mathrm{He}^{2+}$. We have performed a dedicated discharge during a helium campaign at AUG in order to obtain such measurements and to study the behaviour of the ion heat transport in helium. The discharge was carried out with $\mathrm{B}_{t}=-2.5 \mathrm{~T}, \mathrm{I}_{p}=1 \mathrm{MA}$, an average triangularity of $0.28,4.5 \mathrm{MW}$ of NBI heating and 3.4 MW of ECRH.

In this case, we have analyzed one specific ELM crash instead of combining the data from several successive ELMs, as the ELMs did not have a constant frequency over a long period. The temporal resolution of the fast edge CXRS system was set to $100 \mu \mathrm{s}$ and, as was done before, the measurements were rebinned to $250 \mu$ s to have concurrent measurements with the electron density and temperature. In this discharge, the time resolution of the $n_{e}$ measurements was higher due to the limited signal-to-noise ratio of the lithium beam diagnostic in the helium plasma.

Figure 6 shows the pre-ELM profiles of the (a) $T_{e}$, (b) $n_{e}$, (c) $T_{i}$ and (d) toroidal rotation profiles. Here, the ion temperature and toroidal rotation were measured with two core diagnostics (CER and CAR) and two edge systems (CMR and CNR), all measuring on the same beam. In this discharge $N_{2}$ seeding was applied to have an impurity measurement and to study the effect of impurity seeding [39] in helium. Thus, one of the core (CER) and one of the edge (CMR) systems was set to a nitrogen spectral line, $\mathrm{N}^{7+}(n=9 \rightarrow 8)$ at $566.937 \mathrm{~nm}$. As shown in figure 6 the edge main ion (blue) and impurity (red) temperature are approximately the same, confirming a good coupling between main ions and impurities. It should be noted that for the analysis at the plasma edge, the helium plume effect $[40,41]$ has not been taken into account. While the helium plume can affect the plasma core quite substantially [41], the impact on the plasma edge is predicted to be small [40]. Figure 6(d) shows that the difference between main ion and impurity toroidal rotation at the plasma edge can be up to $15 \mathrm{~km} / \mathrm{s}$. While the impurity rotation has a well-like feature $[42,43]$, the main ion rotation has a much less pronounced dip and drops to an almost constant level of $20 \mathrm{~km} / \mathrm{s}$ in the pedestal, indicating a non-negligible source term at the plasma edge. Figure 6(d) also shows the neoclassically predicted main ion toroidal rotation in black. The neoclassical prediction is calculated using the measured electron density and temperature profiles, ion temperature and impurity toroidal rotation profiles and assuming neoclassical poloidal rotation profiles for both main ions and impurities. Previous experiments at AUG showed that the measured poloidal rotation profiles in the plasma edge are in good agreement with neoclassical theory [44]. As shown in figure 6(d), the neoclassical main ion toroidal rotation describes the experimental profile within the uncertainties.

In order to analyze the edge ion heat transport, the core and edge profiles were combined as was done in section 5.1. Figure 7 shows the evolution of (a) the measured 

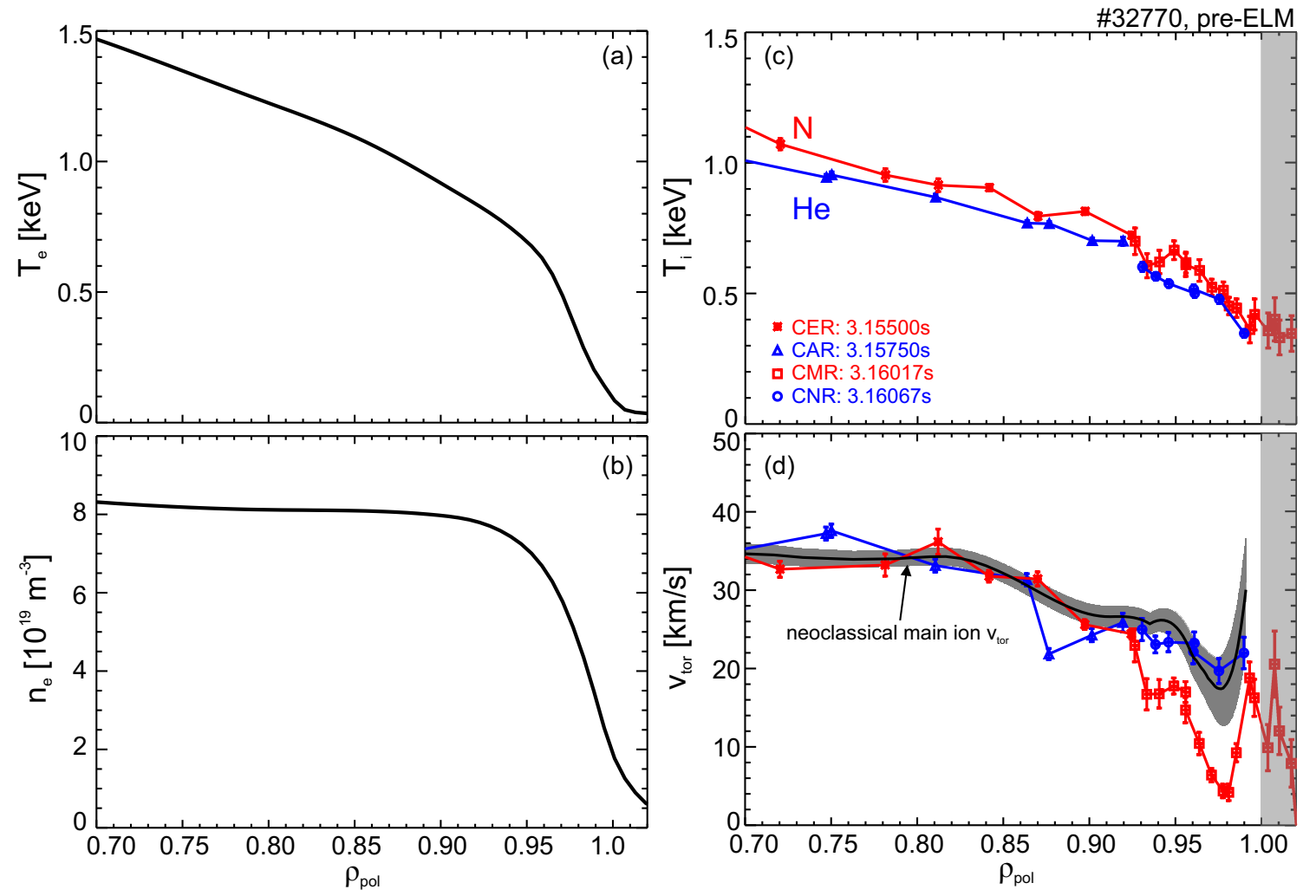

Figure 6. Electron, main ion (He, blue) and impurity (N, red) profiles: (a) $T_{e}$, (b) $n_{e},(\mathrm{c}) T_{i}$ and (d) toroidal rotation profile. The neoclassical prediction of the main ion toroidal rotation is shown in black. The region highlighted in grey marks the scrape-off layer where the CXRS measurements are not reliable due to low signal-to-noise ratio.

ion temperature profiles at fixed $\rho_{\text {pol }}$ values, (b) the ion heat transport coefficient as determined by power balance normalized to the neoclassical prediction and (c) the thermo-currents of the inner and outer divertor, which are used as ELM indicators. Similar to deuterium plasmas, a local increase of $T_{i}$ close to the separatrix is observed at the ELM onset. Note that in the helium discharge the radial coverage of the fast edge CXRS system extended further inside, covering the pedestal from $\rho_{\text {pol }}=0.95$ to the separatrix. As shown in figure 7 (b), the ion heat transport is close to the neoclassical level before the ELM crash in the region where the ion temperature gradient is maximal $\left(0.97<\rho_{\text {pol }}<1.0\right)$. Further inward, the ion heat transport is a factor of $4-5$ above the neoclassical level. During the ELM crash, a heat pulse propagates outwards and the heat transport level is highly anomalous. As was seen in the the deuterium discharge, the ion heat transport and the ion temperature gradient recover 3-4 ms after the onset of the ELM. At the top of the pedestal the ion heat diffusivity is large, while in the maximum gradient region and in the bottom region the ion heat transport can be approximated by the neoclassical value. 


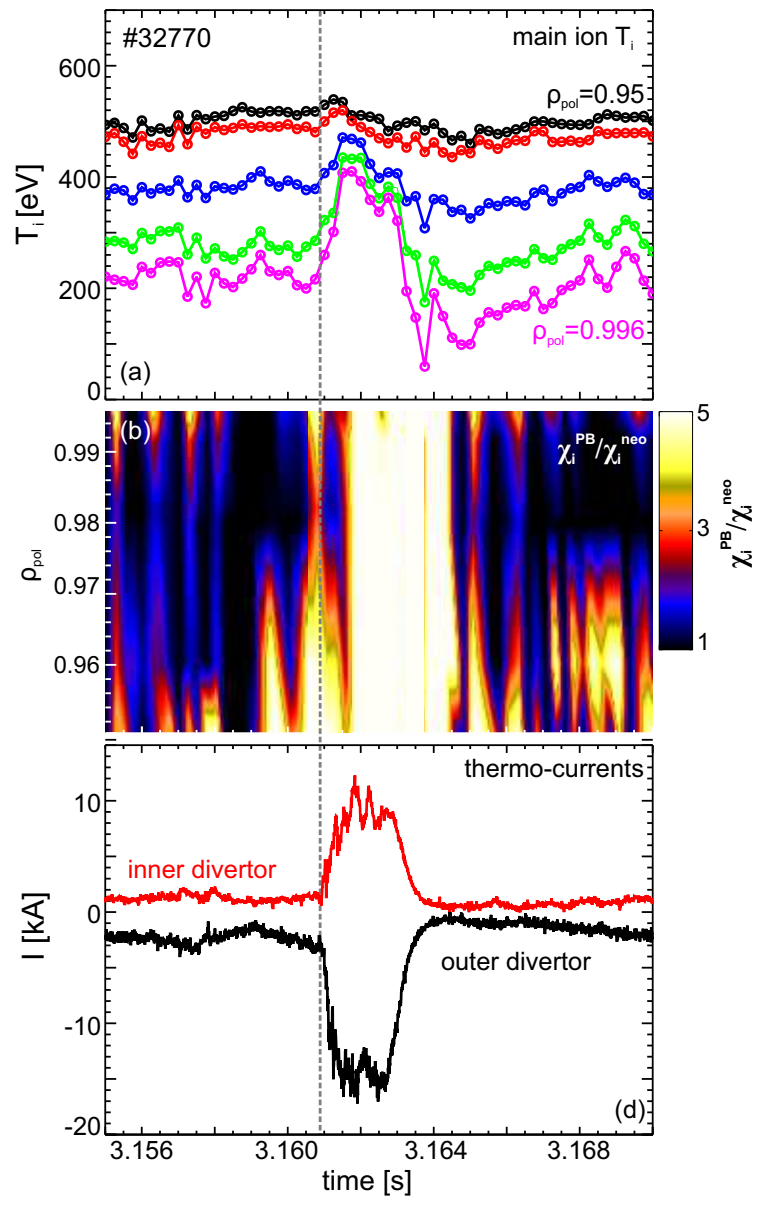

Figure 7. Evolution of the (a) ion temperature, (b) the ratio of the experimental and neoclassical ion heat diffusivity. (c) The thermo-currents of the inner and outer divertor.

\section{Summary}

Pedestal matching experiments in deuterium and hydrogen at AUG reveal that the inter-ELM ion heat transport is close to the neoclassical value, consistent with previous observations in deuterium. The additional power needed in $\mathrm{H}$ to match the $\mathrm{D}$ pedestal temperature mainly flows through the electron channel, i.e. $\chi_{e}$ increases while $\chi_{i}$ stays at the same level within the uncertainties.

Charge exchange measurements with unprecedented time resolution at AUG revealed the dynamics of $T_{i}$ during an ELM [26]. Using these measurements, the ion heat transport was analyzed during the entire ELM cycle. At the ELM onset the ion heat transport increases to strongly anomalous values. The separatrix $T_{i}$ is observed to increase, leading to a reduced $T_{i}$ gradient in the pedestal, and thus, the perturbed ion heat flux increases first at the very plasma edge. Subsequently, the whole pedestal profile reduces during the ELM crash. The build-up of the $T_{i}$ pedestal and the restoration of the ion heat transport to the pre-ELM neoclassical values is obtained 3-4 ms after the ELM. The ELM dynamics of the ion heat transport channel was simulated with a simple 
model in which $\chi_{i}$ was set ad-hoc to high values during the ELM crash to recover the behaviour of $T_{i}$ during the crash and to set the correct starting conditions for the postELM evolution. Using this ansatz, the dynamic behaviour of $T_{i}$ before the ELM and during the recovery phase after the crash could be reproduced with a neoclassical $\chi_{i}$ at the edge.

The ion temperature was also measured in a helium plasma, which enables the measurement of the main ion species by utilizing CXRS on $\mathrm{He}^{2+}$. In this case, the measurements covered a larger radial region at the edge. The ion heat diffusivity is close to the neoclassical level in the steep gradient region, where the ion temperature gradient is maximal, consistent with the observations in D and H. Further inwards $\left(\rho_{\text {pol }} \leq 0.97\right)$ the ion heat diffusivity exceeds the neoclassical transport by a factor of 4-5. The profile evolution during the ELM cycle shows the same behaviour as in D, first the separatrix temperature increases and thus, the gradient in $T_{i}$ is decreased, followed by a subsequent restoration to the pre-ELM profile. While the edge ion heat transport resembles neoclassical values before the ELM crash and during the build-up phase, the ion heat diffusivity during the ELM is highly anomalous and requires non-linear MHD modelling to shed light on the underlying physics during the crash.

\section{Acknowledgments}

The first author would like to thank A. V. Chankin for fruitful discussions and the referees for their valuable input. This work has been carried out within the framework of the EUROfusion Consortium and has received funding from the Euratom research and training programme 2014-2018 under grant agreement number 633053. The views and opinions expressed herein do not necessarily reflect those of the European Commission. The support from the EUROfusion Researcher Fellowship programme under grant number WP14-FRF-IPP/Viezzer and from the Spanish Ministry of Economy and Competitiveness (Grant No. FJCI-201422139) is gratefully acknowledged.

\section{References}

[1] H. Biglari et al. Phys. Fluids B, 2:1, 1990.

[2] P. Gohil et al. Nucl. Fusion, 38(1):93, 1998.

[3] F. Ryter et al. Plasma Phys. Control. Fusion, 43(A323), 2001.

[4] P. Mantica et al. Phys. Rev. Lett., 102:175002, 2005.

[5] M. Kotschenreuther et al. Phys. Plasmas, 2:2381, 1995.

[6] A. M. Dimits et al. Phys. Plasmas, 7:969, 2000.

[7] E. Wolfrum et al. Nucl. Fusion, 55:053017, 2015.

[8] E. Viezzer et al. Nucl. Fusion, 57:022020, 2017.

[9] A. V. Chankin et al. Plasma Phys. Control. Fusion, 48:839, 2006.

[10] H. Urano et al. Phys. Rev. Lett., 95:035003, 2005.

[11] N. A. Pablant et al. Plasma Phys. Control. Fusion, 58:045004, 2016.

[12] A. G. Peeters. Phys. Plasmas, 7(1):268, 2000.

[13] M. Bessenrodt-Weberpals et al. Nucl. Fusion, 33:1205, 1993.

[14] J. Q. Dong et al. Phys. Plasmas, 1:3635, 1994. 
[15] F. Ryter et al. Plasma Phys. Control. Fusion, 58:014007, 2016.

[16] F. M. Laggner et al. Phys. Plasmas, 24:056105, 2017.

[17] H. Urano et al. Nucl. Fusion, 53:083003, 2013.

[18] C. Maggi et al. (submitted), 0:0, 2017.

[19] M. Cavedon et al. Rev. Sci. Instrum., 88:043103, 2017.

[20] A. Burckhart et al. Plasma Phys. Control. Fusion, 52:105010, 2010.

[21] A. Diallo. Phys. Rev. Lett., 112:115001, 2014.

[22] F. M. Laggner. Plasma Phys. Control. Fusion, 58:065005, 2016.

[23] E. Viezzer et al. Rev. Sci. Instrum., 83:103501, 2012.

[24] R. M. McDermott et al. Rev. Sci. Instrum., 88:073508, 2017.

[25] M. Cavedon et al. Nucl. Fusion, 57:014002, 2017.

[26] M. Cavedon et al. Plasma Phys. Control. Fusion, 59:105007, 2017.

[27] W. Suttrop et al. Plasma Phys. Control. Fusion, 39:2051, 1997.

[28] B. Kurzan et al. Rev. Sci. Instrum., 82:103501, 2011.

[29] M. Willensdorfer et al. Plasma Phys. Control. Fusion, 56:025008, 2014.

[30] A. Mlynek et al. Rev. Sci. Instrum., 81:033507, 2010.

[31] R. Fischer et al. Fus. Sci. Technol., 58:675, 2010.

[32] E. Wolfrum et al. Conf. Proceedings of the $22^{\text {nd }}$ IAEA Fusion Energy Conference, EX/P3-7, Geneva, 2008.

[33] J. Neuhauser et al. Plasma Phys. Control. Fusion, 44:855, 2002.

[34] E. Viezzer et al. Nucl. Fusion, 53:053005, 2013.

[35] G. V. Pereverzev et al. IPP Report (5/98), 2002.

[36] P. Helander and D. J. Sigmar. Collisional Transport in Magnetized Plasmas. $1^{\text {st }}$ edition, ISBN 0-521-80798 -0, Cambridge University Press, Cambridge, 2002.

[37] P. A. Schneider et al. Nucl. Fusion, 57:066003, 2017.

[38] M. R. Wade et al. Phys. Plasmas, 12:056120, 2005.

[39] M. Dunne et al. Plasma Phys. Control. Fusion, 59:025010, 2017.

[40] R. J. Fonck et al. Phys. Rev. A, 29(6), 1984.

[41] A. Kappatou et al. (submitted), 0:0, 2017.

[42] T. Pütterich et al. Phys. Rev. Lett., 102(025001), 2009.

[43] E. Viezzer et al. Plasma Phys. Control. Fusion, 55:124037, 2013.

[44] E. Viezzer et al. Nucl. Fusion, 55:123002, 2015. 